\title{
ESTUDIO COMPLETO DE 235 ABORTOS
}

\author{
Doctores Alberto Duarte Contreras * Ramiro Zúñiga Gamboa**, \\ Gonzalo Uribe Botero***, María Josefa Caro Acevedo ****
}

\section{INTRODUCCION}

Sobre un total de 1.055 partos atendidos en el Servicio de Maternidad del Hospital San Juan de Dios, de Cúcuta, entre el $1^{\text {o }}$ de diciembre de 1962 y el 31 de julio del presente año, hubo 267 diagnósticos clínicos de aborto incompleto.

Nos había llamado poderosamente la atención la cifra tan elevada de diagnósticos de aborto hechos en el servicio, y aun cuando sabemos que estos

* Médico Jefe del Departamento de Obstetricia y Ginecología del Hospital San Juan de Dios, de Cúcuta.

** Médico Adjunto del Servicio de Ginecología del Hospital San Juan de Dios, de Cúcuta.

*** Médico Patólogo del Hospital San Juan de Dios, de Cúcuta.

**** Médico Residente del Servicio de Maternidad del Hospital San Juan de Dios, de Cúcuta. ingresos no corresponden ni permiten valorar la frecuencia total de los abortos, nos proponemos analizar este problema haciendo en la totalidad de los casos estudio histopatológico.

Muchos abortos aparecen sin causa determinada; en otros su causa reside en anormalidades del huevo; otros son provocados o se deben a enfermedades intercurrentes. Lo cierto es que ante la multiplicidad de causas y el origen tan incierto de éstas, nos es absolutamente imposible sacar conclusiones del estudio de este problema sin el examen anatomopatológico de todos los casos.

De los 267 raspados uterinos practicados con diagnóstico de aborto incompleto, solamente fueron confirmados 235 con el examen anatomopatológico; ello nos da un margen de error del $11.24 \%$.

Para hacer el diagnóstico de aborto tomamos en consideración la historia clínica, la exploración física, exámenes de laboratorio y el estudio histológico. 
De acuerdo a lo anterior los clasificamos en cinco grupos:

\begin{tabular}{lrr}
\hline & No & $\%$ \\
\hline Sin causa aparente & 203 & 86.5 \\
Aborto habitual & 17 & 7.3 \\
Con antecedentes traumático & 11 & 7.3 \\
Aborto molar & 2 & 8.0 \\
Aborto provocado & 2 & 0.8 \\
\hline \hline
\end{tabular}

\section{RESULTADOS}

Para exponer lós resultados del presente trabajo los hemos dividido en cuatro grupos:
A) Antecedentes.
B) Estado actual.
C) Estado sicosociológico.
D) Resultados anatomopatológicos.

\section{A) ANTECEDENTES. \\ 1. EDAD.}

\begin{tabular}{lcr}
\hline Años & Casos & \multicolumn{1}{c}{ \% } \\
\hline 41 o más & 15 & 6.3 \\
15 a 20 & 36 & 15.3 \\
21 a 25 & 62 & 26.4 \\
26 a 30 & 60 & 25.6 \\
31 a 35 & 39 & 16.7 \\
36 a 40 & 23 & 9.7 \\
\hline \hline
\end{tabular}

La mayor frecuencia la tuvimos entre $\operatorname{los} 21$ y 30 años. La paciente de menor edad fue de 15 años, y la de mayor edad, de 45. El promedio en los
235 casos fue de 27.6 años. El hecho de que más del $50 \%$ de las pacientes fluctuasen entre 21 y 30 años de edad es perfectamente explicable por la mayor actividad sexual en estas edades.

\section{DIETA.}

El régimen alimenticio en estas pacientes, en general, era deficiente; algunas veces en cantidad y siempre en calidad, encontrándose un recargo en hidratos de carbono con deficiencia en proteínas y algunas veces en grasas. La inmensa mayoría presentaba signos de desnutrición y anemia, factor muy importante como coadyuvante en el desencadenamiento del aborto.

3. ANTECEDENTES QUIRURGICOS.

\begin{tabular}{lrr}
\hline Intervención & Casos & \% \\
\hline Raspados uterinos & 32 & 13.6 \\
Cesáreas & 7 & 2.9 \\
Apendicectomía & 6 & 2.5 \\
Herniorrafia & 2 & 0.8 \\
Salpingectomía & 2 & 0.8 \\
Safenectomía & 1 & 0.4 \\
Corrección cistocele & 1 & 0.4 \\
Corrección prolapso & & \\
genital grado III & 1 & 0.4 \\
Tiroidectomía & 1 & 0.4 \\
Colecistectomía & 1 & 0.4 \\
SIN INTERVENCION & 181 & 77.4 \\
\hline \hline
\end{tabular}

El antecedente quirúrgico que observamos con mayor frecuencia fue el raspado uterino anterior, la cesárea y la apendicectomía.

No creemos en que los antecedentes quirúrgicos mencionados tengan relación alguna en la etiología del aborto, 
excepto los raspados uterinos mal hechos que lesionan el orificio interno.

\section{ANTECEDENTES GINECO-} OBSTETRICOS

a) GRAVIDEZ

\begin{tabular}{ccr}
\hline $\begin{array}{c}\text { Embara- } \\
\text { zo actual }\end{array}$ & Casos & $\%$ \\
\hline 1 & 13 & 5.5 \\
2 & 29 & 12.4 \\
3 & 25 & 10.6 \\
4 & 30 & 12.7 \\
5 & 20 & 8.5 \\
6 & 22 & 9.4 \\
7 & 12 & 5.2 \\
8 & 17 & 7.3 \\
9 & 18 & 7.6 \\
10 o más & 47 & 20.0 \\
Sin dato & 2 & 0.8 \\
& 235 & 100.0 \\
\hline
\end{tabular}

La frecuencia mayor de abortos la encontramos en pacientes grávidas, diez o más, siguiéndola mujeres grávidas, dos a cuatro.

Juzgamos que la mayor frecuencia de abortos en las grandes multíparas se deba a la fibrosis uterina o a deficiencias del útero, que traen como consecuencia implantaciones viciosas o deficiencias nutricionales en el embrión. Además, hay que tener en cuenta que dadas las condiciones socioeconómicas de estas pacientes, se aumentan con el número de hijos el rechazo a las nuevas gestaciones $\mathrm{y}$, por lo tanto, los mecanismos abortivos.

0.8 b) ABORTOS ANTERIORES

\begin{tabular}{crr}
\hline $\begin{array}{c}\text { Abortos } \\
\text { ante- } \\
\text { riores }\end{array}$ & Casos & $\%$ \\
\hline Sin & 144 & 61.3 \\
1 & 52 & 22.1 \\
2 & 18 & 7.6 \\
3 & 12 & 5.3 \\
4 o más & 8 & 3.3 \\
Sin dato & 1 & 0.4 \\
& 235 & 100.0 \\
\hline
\end{tabular}

La mayor frecuencia de abortos la encontramos en pacientes sin antecedentes de interrupción de gestaciones an8.5 teriores, seguida de pacientes con un 9.4 aborto previo, lo que está en desacuer5.2 do con la teoría de Malpas, aparecida

.3 en 1938 , que pretendía probar que au-

7.6 mentaban las posibilidades de aborto 0.0 con el número de abortos anteriores.

c) MENARQUIA

La edad promedio de la menarquía en las pacientes de estudio fue de 13.4 años, habiéndose encontrado la mayor frecuencia de abortos en aquelias mujeres cuya primera menstruación apareció a los 14 años de edad.

\begin{tabular}{ccr}
\hline Edad & Casos & $\%$ \\
\hline 10 & 2 & 0.8 \\
11 & 3 & 1.2 \\
12 & 27 & 11.6 \\
13 & 48 & 20.4 \\
14 & 73 & 31.2 \\
15 & 46 & 19.6 \\
16 & 17 & 7.3 \\
17 & 9 & 3.8 \\
18 & 2 & 0.8 \\
Sin dato & 8 & 3.4 \\
& 235 & 100.0 \\
\hline
\end{tabular}


Los datos obtenidos respecto a la menarquía, en relación con la mayor frecuencia de abortos, comparándolos con los resultados encontrados en una encuesta, a 500 pacientes que presentaron partos a término en el Servicio de Maternidad del Hospital San Juan de Dios, de Cúcuta, no reveló valor alguno respecto a la edad de la aparición de la primera menstruación, ya que el promedio de edad en éstas fue de 13.7 años y el promedio de edad de las 235 pacientes que estamos estudiando fue de 13.4 años.

B) ESTADO ACTUAL

\section{PAtologia, Ginecologia}

\begin{tabular}{lrr}
\hline Examen ginecológ. & Casos & $\%$ \\
\hline Cervicitis & 12 & \multicolumn{1}{c}{5} \\
Retroversoflexión & 10 & 4.2 \\
Anteversión & 8 & 3.3 \\
Látero desviaciones & 8 & 3.3 \\
Desgarros del cérvix & 8 & 3.3 \\
$\begin{array}{l}\text { Desgarro perineal } \\
\text { antiguo }\end{array}$ & 2 & \\
Prolapso genital & & 0.8 \\
grado II & 1 & \\
Amibiasis del cérvix & 1 & 0.4 \\
Sin patología & 185 & 1.4 \\
& 235 & 100.0 \\
\hline
\end{tabular}

La mayor frecuencia de abortos en pacientes c o n patología ginecológica asociada se encontró en aquellas que presentaban cervicitis, seguida de las malposiciones uterinas.

Creemos que el factor infección y la retroversoflexión fija desempeñan papel importante en la interrupción de la gestación.

\section{EDAD DEL EMBARAZO}

\begin{tabular}{ccc}
\hline Meses & Casos & \% \\
\hline 1 & 17 & 7.4 \\
$11 / 2$ & 45 & 19.2 \\
2 & 52 & 22.1 \\
$21 / 2$ & 39 & 16.6 \\
3 & 52 & 22.1 \\
$31 / 2$ & 2 & 0.8 \\
4 & 14 & 6 \\
$41 / 2$ & 1 & 0.4 \\
5 & 3 & 1.2 \\
Sin dato & 10 & 4.2 \\
& 235 & 100.0 \\
\hline
\end{tabular}

La mayor frecuencia de abortos se presentó en embarazos entre uno y medio y tres meses. En nuestra opinión, la mayor frecuencia del aborto en estas edades del embarazo están muy de acuerdo con los factores ovulares y maternos del aborto, excepción hecha de la incompetencia del cuello uterino.

\section{PATOLOGIA CONCOMITANTE}

\begin{tabular}{lrr}
\hline Enfermedad & Casos & $\%$ \\
\hline $\begin{array}{lr}\text { Várices miembros } \\
\text { inferiores }\end{array}$ & 211 & \\
Parasitismo intesti- & & 89.6 \\
nal y anemia & 210 & 89.2 \\
Infec. urinarias & 23 & 9.7 \\
Colecistitis & 2 & 0.8 \\
Paludismo evolutivo & 1 & 0.4 \\
Enfermedad mental & 1 & 0.4 \\
\hline
\end{tabular}

Entre las enfermedades concomitantes que presentaron estas pacientes se encuentran el parasitismo intestinal asociado con la anemia y las grandes várices de miembros inferiores.

Es de todos bien conocido que el parasitismo intestinal inferioriza nota- 
blemente el organismo femenino para tolerar la sobrecarga que significa el embarazo, y en nuestro concepto es la causa más frecuente, junto con las carencias nutricionales asociadas, de la mayoría de los abortos espontáneos.

\section{SINTOMA PREDOMINANTE}

\begin{tabular}{lrr}
\hline \hline Síntoma & Casos & $\%$ \\
\hline Hemorragia & 54 & 23 \\
Dolor & 1 & 0.4 \\
$\begin{array}{l}\text { Hemorragia y dolor } \\
\text { Hemorragia- }\end{array}$ & 168 & 71.4 \\
dolor-fiebre & 12 & 5.2 \\
& 235 & 100.0 \\
\hline
\end{tabular}

Los síntomas más frecuentes encontrados en estas pacientes fueron la hemorragia y el dolor asociados.

C) ESTUdio SICOLOGICO.

\begin{tabular}{lcc}
\hline Estado civil & Casos & $\%$ \\
\hline Solteras & 99 & 42.2 \\
Unión ilegal & 72 & 30.7 \\
Casadas & 52 & 22.1 \\
Abandonadas & 8 & 3.3 \\
Viudas & 4 & 1.7 \\
& 235 & 100.0 \\
\hline
\end{tabular}

Al considerar el estado civil de las pacientes encontramos que la mayoría eran solteras, seguidas con un margen muy estrecho por las que vivían en unión ilegal. Esto tiene una gran importancia en la etiología del aborto y en la frecuencia del aborto provocado.

Respecto al grado de instrucción, la mayoría de las pacientes solamente tenían estudio de enseñanza primaria, y de ellas únicamente un pequeño grupo terminó la instrucción escolar; también había un buen número de analfabetas.
Las Condiciones económicas de la generalidad de los hogares de estas pacientes eran muy precarias. Más del $60 \%$ de las pacientes no deseaban tener más hijos debido, según ellas, a las malas condiciones financieras de sus hogares.

\section{D) RESULTADOS}

\section{ANATOMOPATOLOGICOS}

En todos los casos, el estudio anatomopatólico confirmó el diagnóstico de aborto con el hallazgo de vellosidades coriales y los casos en que no se las pudo encontrar, aunque se hubiera observado reacción decidual franca, se excluyeron de este estudio, salvo aquellos casos en que se encontró tejido trofoblástico o el signo de Arias Stella en el endometrio.

Se consideran en este trabajo solamente las pacientes que presentaron aborto comprobado por el estudio histopatológico.

Estudiamos los hallazgos histopatológicos en:

$1^{\text {o }}$ Vellosidades coriales.

20 Decidua.

3o Endometrio.

40 Varios.

19 VELLOSIDADES CORIALES

\begin{tabular}{lcr}
\hline Hallazgos & Casos & \% \\
\hline Jóvenes: & & \\
Primer trimestre & 60 & 58.2 \\
Segundo trimestre & 35 & 33.9 \\
Adultas: & & \\
Degener. hialina & 44 & 42.7 \\
Degener. hidrópica & 19 & 18.4 \\
Necrosis & 48 & 46.6 \\
Fibrosis & 27 & $\mathbf{2 6 . 2}$ \\
Eritroblastos & 4 & 3.9 \\
\hline
\end{tabular}


Estudiamos 235 casos de raspados uterinos fijados en formol al $10 \%$, incluídos en parafina y coloreados con hematoxilina-eosina. De ellos sólo alcanzamos a revisar 143 , o sea el $60.7 \%$ de los casos del presente trabajo.

En 103 muestras encontramos vellosidades coriales: 95 jóvenes, $60 \mathrm{del}$ primer trimestre y 35 del segundo trimestre; 8 fueron de tipo adulto. No hubo desacuerdo entre el tiempo de embarazo y su aspecto histológico.

Los cambios estudiados en orden de frecuencia fueron: necrosis en 48 casos, degeneración hialina en 44 , degeneración hiadrópica en 19 y fibrosis en 27. En 4 casos nos llamó especialmente la atención la existencia de abundantes eritroblastos que surgieron la posibilidad diagnóstica de eritroblastosis.

Un aspecto que juzgamos de interés, relacionado con la inflamación, es lo poco que son atacadas las vellocidades coriales por el proceso inflamatorio; solamente en pocos casos que no contabilizamos se apreció claramente el infiltrado en su estroma.

En cuanto a otros trastornos como degeneración hialiana, degeneración hidrópica, etc., que presentaban las vellosidades coriales, pueden estar íntimamente relacionados con el cambio vascular, responsable de una mala irrigación.

\section{2\% DECIDUA}

\begin{tabular}{lcc}
\hline Hallazgos & \multicolumn{1}{c}{ Casos } & $\%$ \\
\hline Inflamación aguda & 62 & 48.8 \\
Inflamación crónica & 52 & 40.9 \\
Degeneración hialina & 71 & 55.9 \\
Necrosis & 101 & 79.5 \\
Hemorragia & 94 & 74 \\
\hline
\end{tabular}

En 127 casos encontramos tejido decidual $(88.8 \%)$, cuyos cambios en orden de frecuencia fueron: necrosis en 101 casos, hemorragia en 94, degeneración hialina en 71 , inflamación aguda en 62 , inflamación crónica en 52 , ligera en dos casos.

El criterio seguido para clasificar la inflamación fue la mayor intensidad del infiltrado leucocitario; el predominio polimorfonuclear para los casos agudos, y la mayor intensidad inflamatoria con predominio de mononucleares en los casos crónicos. En algunos casos, como en el señalado como M218 de nuestro laboratorio, se observó presencia de células gigantes, que creemos se deriven del sincitiotrofoblasto, que según los estudios recientes de Pierce (3) participan de la secreción de hormona gonadotrófica, en oposición a lo que siempre se ha dicho que es secretada por el citotrofoblasto.

Nos llamó la atención en este estudio los notorios cambios inflamatorios, que difícilmente podemos clasificar como primarios o secundarios al aborto. Se podría pensar de acuerdo con lo frecuente que se presentan los cambios necróticos, que en su producción juega papel especial el engrosamiento de la pared vascular con trombosis en muchos casos. Luego es más fácil interpretar los cambios inflamatorios como secundarios. Nos quedaría por explicar el origen del trastorno vascular.

\section{3ํ ENDOMETRIO}

\begin{tabular}{lcc}
\hline Hallazgos & Casos & $\%$ \\
\hline Signo Arias Stella & 19 & 20 \\
Inflamación aguda & 22 & 23.1 \\
Luteínico & 45 & 47.3 \\
Inflamación crónica & 9 & 9.4 \\
\hline
\end{tabular}


Observamos en 45 casos la presencia de tejido endometrial de tipo luteínico, 22 con inflamación aguda y 9 con inflamación crónica. En 19 casos el signo de Arias Stella (4), o sea la presencia de hipertrofia e hipercromasía nuclear de numerosos núcleos, así como la reduplicación celular y extrema vacuolización, fue positivo.

\section{3ํ VASOS}

\begin{tabular}{lcr}
\hline Hallazgos & Casos & $\%$ \\
\hline Gruesos hialinos & 53 & 65.4 \\
Trombosis & 21 & 25.9 \\
Fibrosis & 7 & 8.6 \\
\hline
\end{tabular}

En estos casos nos llamó la atención la existencia de marcado engrosamiento de su pared, con hialinización en 53 casos, trombosis muy evidente en 21 casos, fibrosis de la pared en 7 y proliferación endotelial en muchos de ellos no contabilizados.

Del estudio comparativo entre los cambios placentarios y la edad de las pacientes no se puede concluír que ésta influya o esté relacionada con el cambio vascular, aunque el mayor número (33 casos) se presentó entre los 20 y 29 años.

Pero no dudamos que la esclerosis vascular miometrial es más notoria en pacientes de mayor edad. Tampoco la gravidez tiene relación específica con los cambios placentarios, ya que la mayoría ( 11 casos) correspondían al segundo embarazo.

\section{CONCLUSIONES}

1. El estudio de los raspados uterinos es útil para confirmar o descartar el diagnóstico de aborto.

20 Sirve para el descubrimiento de procesos inflamatorios específicos como la tuberculosis o la sífilis; también en la eritrosblastosis.

$3^{\circ}$ No hay relación evidente entre los trastornos vasculares y la edad y el número de gestaciones en estas pacientes.

49 Los cambios vasculares sugieren la existencia de algún trastorno específico, que debe ser investigado con más cuidado.

50 La trombosis vascular puede explicar en gran número de casos el descubrimiento del proceso abortivo.

69 El signo de Arias Stella es de gran ayuda en el diagnóstico del embarazo, pero ni su presencia lo confirma ni su ausencia lo descarta.

79 No es posible hacer el diagnóstico de aborto criminal por el estudio histológico únicamente.

\section{RESUMEN}

Se estudian 235 historias clínicas de abortos del Servicio de Maternicaad del Hospital San Juan de Dios, de Cúcuta.

Se dan conclusiones. 


\section{BIBLIOGRAFIA}

1. OROPEZA MARTINEZ GERMAN y colaboradores. Aborto incompleto. Correlación clínico-patológica en 100 casos con legrado uterino. Rev. Gin. y Obst. de México. 97: 171188. 1962.

2. ISAZA MEJIA GUSTAVO: Doscientos setenta raspados uterinos para el aborto. Rev. Col. Obst. y Gin. 5: 539-549. 1960.

3. PIERCE G. B. Jr. and MIDGLEY A. R. Jr. The origin and function of human sincytiotrophablactic gigant cells. Amer. J. Path. 2: 153. 1963.

4. ARIAS STELLA J.: Características, significación y patogenia de las alteraciones atípicas endometriales asociadas a la presencia de tejido coriónico. Premio Russel Perú. 1960. 